\title{
O Monitoramento e a Avaliação na Gestão do Ministério da Saúde
}

$O$ processo de monitoramento e avaliação (MEA) das políticas públicas de saúde ocorre, há muitas décadas, na gestão do Sistema Único de Saúde (SUS), por meio da utilização de conceitos, de metodologias e dos mais diversos instrumentos. Esse modus operandi, ao mesmo tempo em que possibilitou a disseminação e o aprofundamento dessa prática em áreas estratégicas, necessita de uma visão mais sistêmica de forma a contribuir com o processo de implementação de políticas e programas.

Diante disso, a criação de um Departamento de Monitoramento e Avaliação do SUS (Demas), no âmbito da Secretaria-Executiva (SE) do Ministério da Saúde (MS), representa uma importante iniciativa. Planejar, avaliar e monitorar são ações essenciais para a consecução das políticas públicas e que ganham potência política na atual gestão do Ministério da Saúde.

O processo de planejamento, monitoramento e avaliação na gestão atual do Ministério da Saúde teve seu início marcado pela necessidade de harmonização de diversos instrumentos: o Plano Plu- rianual (PPA) 2008-2011, que estava terminando; o PPA 2012-2015, que estava começando; o "Mais Saúde", que era o recorte estratégico da gestão anterior; o novo recorte estratégico; o Plano Nacional de Saúde (PNS) 2008-2011; o PNS 2012-2015; e, por fim, a Programação Anual de Saúde (PAS), que contém ações, recursos e outros elementos voltados para o cumprimento das metas do PNS.

Dessa forma, em 2011 o MS se empenhou em um Planejamento Estratégico (PE) para compatibilizar o cerne desses instrumentos. Foram, então, formulados 16 objetivos estratégicos, que, como norteadores institucionais, passaram a fundamentar a visão de futuro do MS para a gestão de 2011 a 2014 .

Destes 16 objetivos, 15 estão diretamente relacionados à gestão da saúde, enquanto o 160, "contribuir para erradicar a extrema pobreza no país", é um objetivo transversal do governo. Assim, o atual Plano Estratégico do MS, com seus vários instrumentos (PPA, PNS, PAS e PE), expressa os compromissos com as necessidades do SUS e com o Plano de Governo. 
- FIGURA 1: RESUMO DOS 16 OBJETIVOS ESTRATÉGICOS DO MINISTÉRIO DA SAÚDE

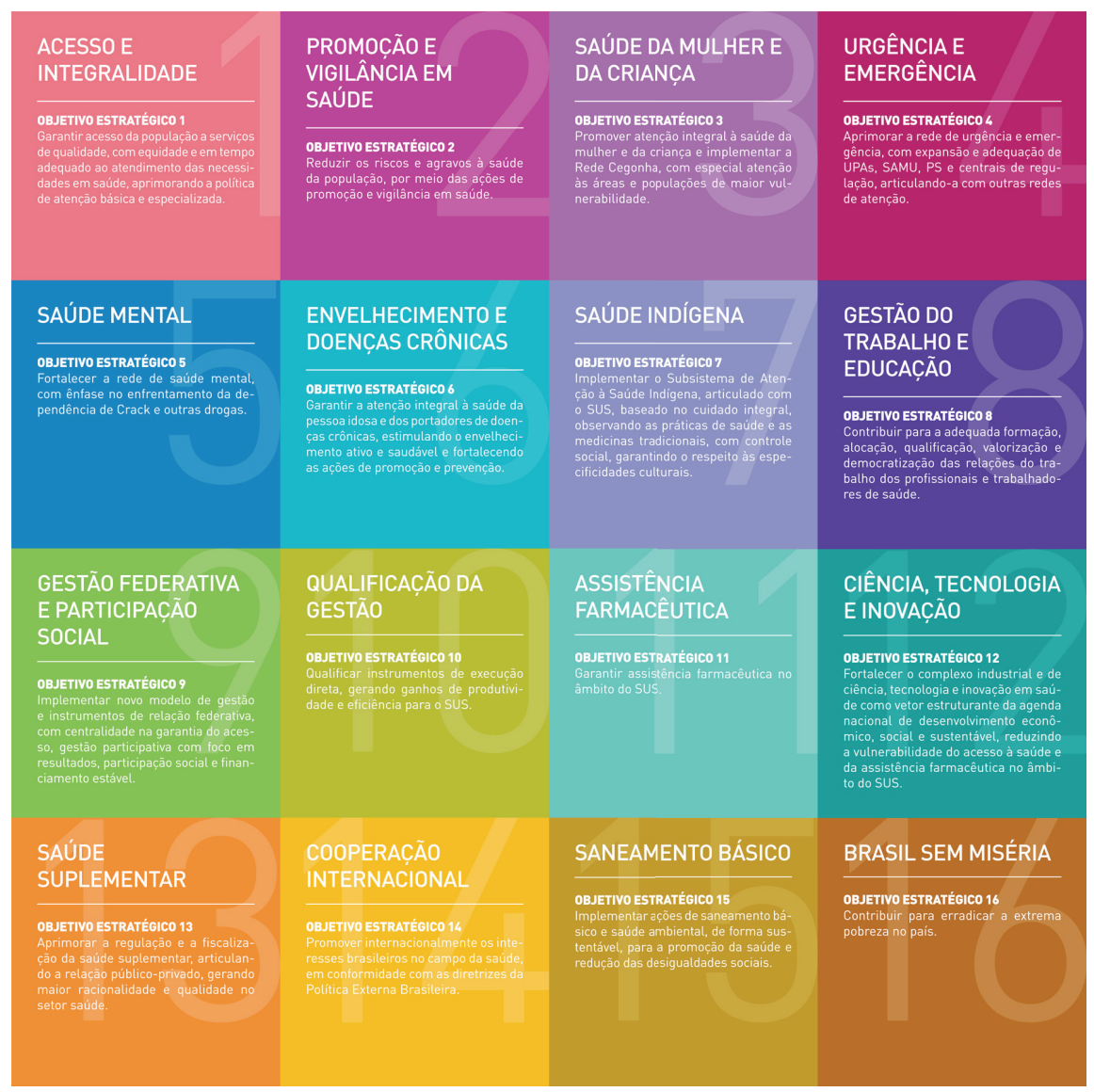

Fonte: Demas/SE/MS

Além do alinhamento estratégico entre PPA, PNS e PAS, o processo de planejamento, monitoramento e avaliação do Ministério da Saúde foi, paulatinamente, sendo aprimorado para proporcionar mais transparência às ações desenvolvidas e para dar visibilidade àquilo que está sendo feito em prol da saúde da população. 0 fluxograma apresentado na Figura 2 é representativo da governança interna e dos espaços decisórios. 


\section{- FIGURA 2: INSTRUMENTOS DE GOVERNANÇA INTERNA DO MINISTÉRIO DA SAÚDE}

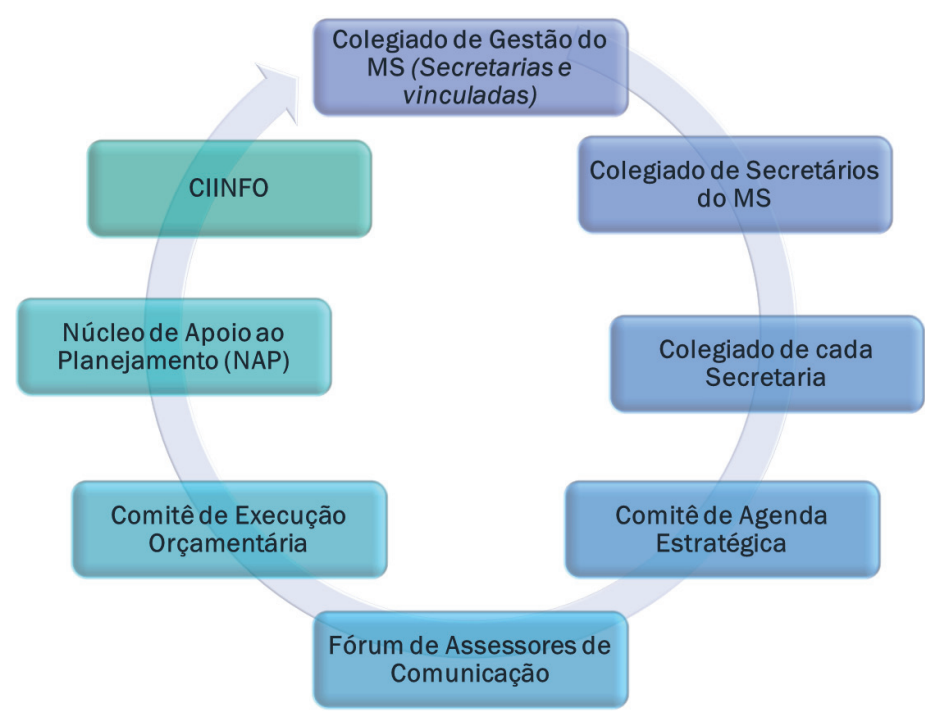

Fonte: Demas/SE/MS

O sucesso de qualquer ação de M\&A depende de um efetivo planejamento e gestão da informação. Seu enraizamento, entretanto, depende, primordialmente, de um acordo prévio, de uma decisão política, de sua adoção como objetivo de governo. Do contrário, mesmo que se faça o planejamento mais apurado e que se desenvolvam as melhores ferramentas, o processo não sobreviverá.

A partir de sua criação, coube ao departamento, por exemplo, outras ações estruturantes, tais como: coordenação da formulação e da implementação da Política de Monitoramento e Avaliação do SUS (em andamento); revisão da Política Nacional de Informação e Informática do SUS (PNIIS) (concluída e pronta para pactuação); e construção de uma estrutura especializada em produção de informações qualificadas para os gestores da saúde (a chamada BI - business intelligence ou inteligência de negócio, ação de fluxo contínuo). Tais incumbências indicam o papel estratégico do Demas na produção e distribuição de informações que contribuam efetivamente para a redução das incertezas no processo decisório do SUS (em geral) e do Ministério da Saúde (em particular). 
Vale ressaltar que o papel do Demas e da Subsecretaria de Planejamento e Orçamento (SPO/SE) se imbricam. Além disso, esse processo só é viável porque há uma rede que se propõe a aprimorá-lo, de forma constante, com participação de todas as áreas do Ministério. Nesse fazer cotidiano são acompanhados, por meio de reuniões e oficinas periódicas, os vários instrumentos já citados (PNS, PPA, PE, PAS), que são subsídios fundamentais para $O$ Relatório de Gestão Anual do Ministério da Saúde e do governo como um todo.

Diante disso, o Departamento direcionou seu trabalho para importantes ações, tais como: (i) a adoção do Sistema Controle, Acompanhamento e Avaliação de Resultados (E-Car), um instrumento de gestão e acompanhamento, amplamente flexível, configurável e de código aberto; (ii) a gestão da informação estratégica em saúde com ênfase na reestruturação da Sala de Apoio à Gestão Estratégica (Sage), um instrumento de produção e disseminação de dados e informações, apto a contribuir para a redução das incertezas do processo decisório no nível estratégico da gestão em saúde; e (iii) a criação do Índice de Desenvolvimento do SUS (IDSUS), um indicador composto, que busca avaliar o desempenho (nos três níveis de gestão: municipal, estadual e federal) do "SUS que atende os residentes de cada município brasileiro".
Controle, Acompanhamento e Avaliação de Resultados (E-Car)

Originalmente desenvolvido por uma cooperação técnica firmada entre o Serviço Federal de Processamento de Dados (Serpro) e a Companhia de Tecnologia da Informação e Comunicação do Paraná (Celepar), o e-Car é uma ferramenta tecnológica voltada para o acompanhamento estratégico e para o gerenciamento operacional de ações. Além de um minucioso monitoramento, o instrumento é um software livre, dispondo de uma estrutura de informação plenamente adaptável e harmonizável com as soluções já existentes no MS.

Essa alta governabilidade sobre a ferramenta possibilita o acompanhamento das ações, de acordo com as periodicidades desejadas, por meio do registro de dados objetivos e subjetivos. Ou seja, é possível inserir metas, indicadores e prazos quantificáveis, mas também pareceres subjetivos com as visões e percepções dos responsáveis por cada umas das ações.

É possível, ainda, a segmentação por tipos de acompanhamento, separando as ações por conjuntos. Dessa forma, um re- 
corte estratégico de resultados pode ser monitorado de forma diferenciada, disponibilizando uma visão gerencial - com os respectivos status (satisfatório, alerta ou crítico) de cada resultado -, por meio da qual é possível comparar períodos acompanhados e avaliar o andamento das ações e a evolução de seus indicadores ao longo do tempo.

Na atual agenda do MS, vinculados aos 16 Objetivos Estratégicos existem 131 estratégias, 601 resultados, 1.365 produtos, 762 ações e 435 atividades. Cada área do MS é responsável pela inserção das informações relacionadas às suas ações de trabalho. A partir disso, foi desenvolvida uma sistemática de monito- ramento com duas periodicidades: mensal e quadrimestral.

De quatro em quatro meses, todos os resultados são averiguados em ciclos de monitoramento que implicam o seguinte fluxo: (i) cada área avalia e atualiza os resultados e indicadores sob sua responsabilidade no e-Car; (ii) os colegiados das secretarias validam os status e identificam os pontos críticos; (iii) o Demas promove oficinas transversais de validação por Objetivos Estratégicos; (iv) o Núcleo de Apoio ao Planejamento (NAP), instância criada para coordenar o processo de planejamento estratégico, analisa o monitoramento realizado; e (v) o Colegiado de Gestão do MS realiza o acompanhamento geral do PE.

\section{- FIGURA 3: TELA INTERNA DO MONITORAMENTO DO PLANO ESTRATÉGICO DO MINISTÉRIO DA SAÚDE}

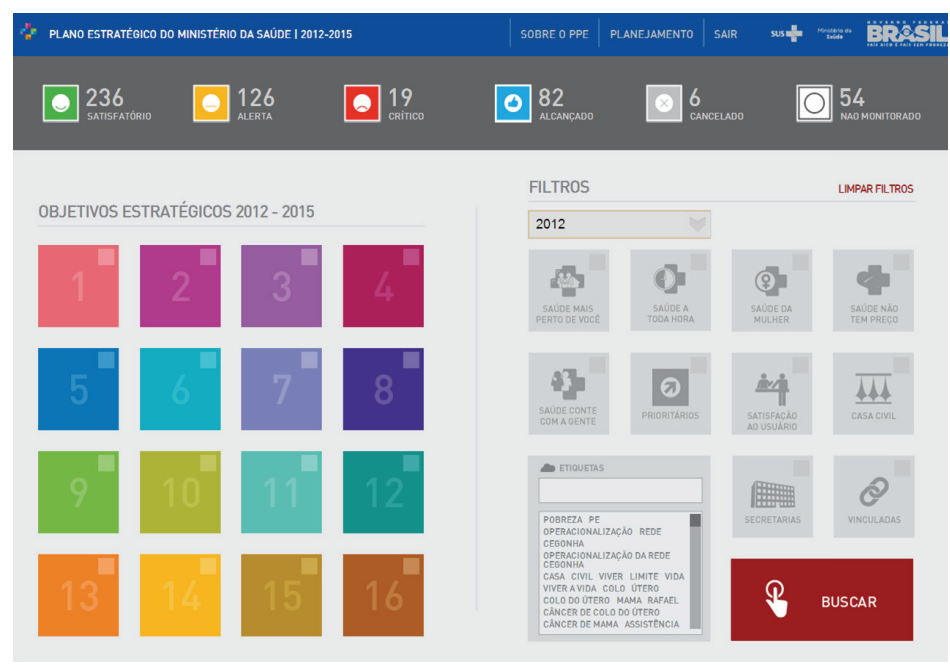


No início de 2012, a revisão do Planejamento Estratégico do MS destacou um elenco de indicadores prioritários para o alcance dos resultados pactuados, cujo acompanhamento periódico, como forma de trazer mais eficácia às ações previstas, deveria ser realizado pelo Ministro da Saúde e seu Colegiado. Esse destaque - no qual se incluem importantes ações como: Rede Cegonha, SOS Emergência, Combate ao Crack e Saúde Não Tem Preço - passou a ser chamado de Recorte Estratégico do Ministério da Saúde (REM).

Por conseguinte, mensalmente os 100 resultados REM são inspecionados, respeitando o seguinte fluxo: (i) cada área avalia e atualiza os REM sob sua responsabilidade no e-Car; (ii) os colegiados das secretarias validam os status e identificam os pontos críticos; (iii) o Núcleo de Apoio ao Planejamento (NAP) analisa o monitoramento realizado; e (iv) o Ministro da Saúde e a Secretária Executiva realizam o acompanhamento executivo do recorte estratégico.

\section{Gestão da informação estratégica em saúde com ênfase na reestruturação da Sala de Apoio à Gestão Estratégica (Sage)}

O Brasil é o único país do mundo com mais de 100 milhões de habitantes que oferece um sistema de saúde público e universal.

\section{FIGURA 4: FLUXO DE MONITORAMENTO OUADRIMESTRAL DOS RESULTADOS DO PLANO ESTRATÉGICO DO MINISTÉRIO DA SAÚDE}
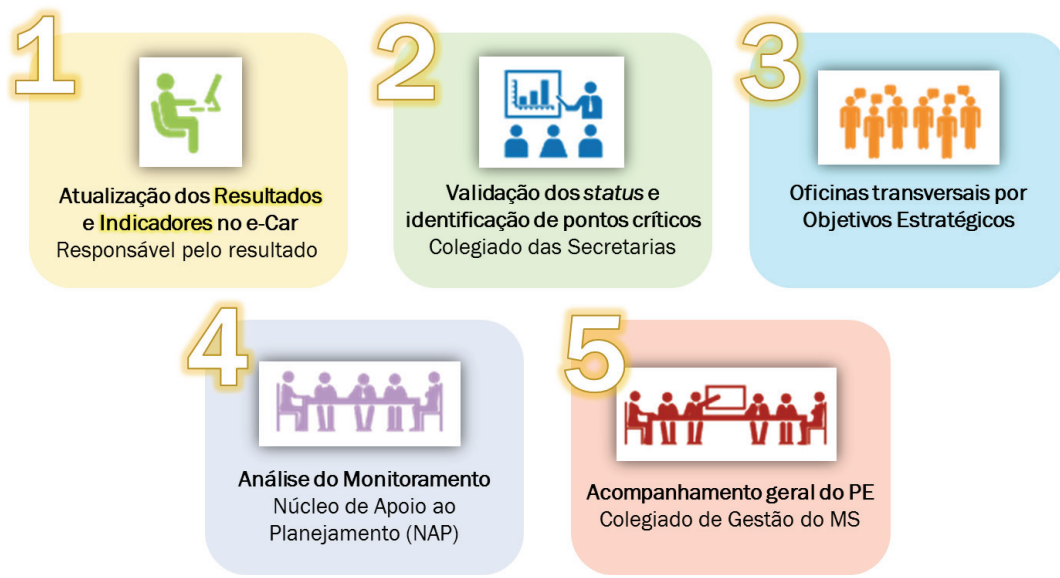

Fonte: Demas/SE/MS 
Ofertar atenção à saúde integral, de qualidade e com acesso amplo para toda a população é um enorme desafio. Anualmente, o Sistema Único de Saúde (SUS) realiza mais de 3 bilhões de procedimentos ambulatoriais, 500 milhões de consultas médicas, 12 milhões de internações e 30 milhões de procedimentos oncológicos. Além disso, o sistema realiza o maior número de transplantes de órgão do mundo e movimenta 90\% do mercado mundial de vacinas.

A informação em saúde tem como esfera de intervenção tanto a consciência subjetiva do cidadão e o exercício do controle social quanto o atendimento às complexas estratégias de decisão do gestor público de saúde, de desenvolvimento tecno-científico e de articulação da saúde com as demais políticas sociais e econômicas do País.
Atento a essas necessidades e considerando que grande parte da atividade da área da saúde está na gestão da informação e que a governança da informação passou a ocupar lugar estratégico no fortalecimento das relações interfederativas e no relacionamento governo-cidadão, o MS redefiniu o Comitê de Informação e Informática em Saúde (CIINFO) em 2011, durante o processo de planejamento e alinhamento estratégico.

Instância de decisão colegiada - com representação de todos os secretários, presidentes das entidades vinculadas ao MS, além de relevantes membros consultivos como os da Rede Interagencial de Informações para a Saúde (RIPSA) e do Ministério do Planejamento -, o CIINFO possui funções diretivas, normativas e fiscalizadoras das atividades relativas aos sistemas de informação e informática em saúde, no âmbito do MS e do SUS.

\section{- FIGURA 5: FLUXO DE MONITORAMENTO MENSAL DO RECORTE ESTRATÉGICO DO MINISTÉRIO DA SAÚDE (REM)}
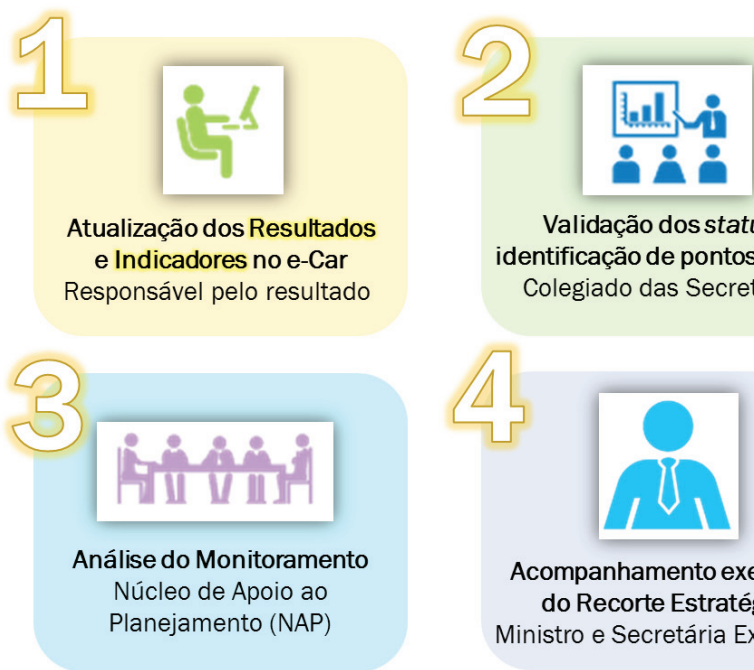

Validação dos status e identificação de pontos críticos Colegiado das Secretarias

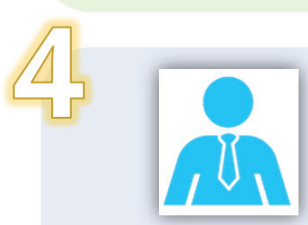

Acompanhamento executivo do Recorte Estratégico Ministro e Secretária Executiva 
A esse Comitê compete, por exemplo, emitir orientações, normas e padrões técnicos de interoperabilidade de informações; promover a racionalização do desenvolvimento e do uso dos recursos de Tecnologia da Informação (TI); e definir padrões essenciais de informação em saúde para suportar um registro eletrônico de saúde, interoperável e compartilhado em todo território nacional.

Uma das deliberações do CIINFO foi promover a reformulação da Política Nacional de Informação Informática em Saúde (PNIIS) visando à melhoria do acesso e da qualidade no SUS; a transparência e segurança das informações; o suporte da informação para tomada de decisão por parte do gestor e profissional de saúde; e, por fim, o desenvolvimento institucional do SUS com ganhos de eficiência em gestão de pessoas, aquisição de insumos, monitoramento e avaliação das ações, logística, pagamento e transferência de recursos.

Originalmente escrita em 2004, a PNIIS necessitava de uma revisão e, principalmente, de uma pactuação tripartite para deixar de ser vista apenas como um documento consultivo. Desse modo, após as sugestões apresentadas em uma consulta pública, a concepção atual da Política procura espelhar a governança descentralizada da informação, passando a vislumbrar a possibilidade de emitir orientações, normas e padrões de interoperabilidade de informações, bem como promover a ra- cionalização do desenvolvimento e do uso dos recursos de Tecnologia da Informação. Atualmente em fase final de elaboração e sistematização das diversas contribuições recebidas, a PNIIS deve ser publicada no $2^{\circ}$ semestre de 2013 após sua aprovação pela Comissão Intergestores Tripartite (CIT).

A nova PNIIS procura atualizar-se a novos conceitos de saúde, como os trazidos pela Lei de Acesso à Informação e pelo uso do E-gov, como uma linha transversal de alinhamento de todas as políticas de informação e informática adotadas pelo Governo Federal. No âmbito dos conceitos mais recentes, ela apresenta, por exemplo, o E-Health, de acordo com o qual o cidadão/paciente deve ter acesso a suas informações pessoais e prontuário para que ele próprio possa decidir, interativamente com o médico e com o gestor, sobre qual é o melhor encaminhamento para seu caso. Além disso, a PNIIS 2013 traz diretrizes importantes como: governança, infraestrutura, conectividade, funcionalidade, segurança e recursos humanos.

Outra importante deliberação do CIINFO foi o recorte e a redefinição do fluxo de informações consideradas estratégicas para o MS. A área da saúde, em suas três esferas de gestão, produz uma vasta quantidade de informações. Por essa razão, é fundamental estabelecer um recorte, um foco para que os gestores possam ter capacidade de monitorar os indicadores mais relevantes e promover discussões para 
modificá-los, tendo em vista a melhoria da saúde da população. Ou seja, não se trata apenas de registrar as diversas informações em uma série histórica, mas possibilitar a interação, a correção do percurso de uma determinada ação ou programa. Em consonância com essa determinação, o Demas envidou esforços e promoveu a reestruturação da Sala de Apoio à Gestão Estratégica do MS (Sage), antiga Sala de Situação em Saúde.

Aberta para o acesso de qualquer cidadão no endereço www.saude.gov.br/sage, a Sala de Apoio à Gestão Estratégica do MS obtém, sistematiza e disponibiliza on line dados e informações produzidas pelo Ministério da Saúde, por entidades vinculadas e por órgãos de pesquisa. Dessa maneira, com cerca de quatro mil visitas diárias, a Sage é um instrumento - de produção, disseminação de dados e informações, gestão e geração de conhecimento - apto a contribuir para a redução das incertezas no processo decisório da gestão em saúde. Além disso, demonstrando a atuação governamental no âmbito do SUS, ela contribui para a transparência das ações desenvolvidas na área da saúde e possibilita projeções e inferências setoriais.

No intuito de organizar os conteúdos e facilitar o acesso e a compreensão dos diversos dados e informações disponibilizadas, a navegação na Sage é atualmente estruturada a partir de cinco módulos: Redes e Programas; Atenção à Saúde; Sociodemográfico; Situação de Saúde; e Gestão/ Financiamento.
- Redes e Programas: Apresenta informações do módulo de "Atenção à Saúde" reagrupadas de acordo com as redes prioritárias de saúde do governo: Saúde Conte com a Gente (saúde mental); Saúde da Mulher; Saúde Mais Perto de Você (atenção básica); Saúde Não Tem Preço (assistência farmacêutica); e Saúde Toda Hora (urgência e emergência).

- Atenção à Saúde: Permite o acompanhamento das metas físicas, das coberturas e dos quantitativos relacionados às principais ações e programas do Ministério da Saúde.

- Sociodemográfico: Apresenta aspectos socioeconômicos, demográficos e territoriais que permitem identificar a situação conjuntural brasileira. Reúne dados políticos de estados e municípios, bem como as representações no Poder Legislativo e sua atuação na área da saúde, por meio de emendas e convênios.

- Situação de Saúde: Disponibiliza indicadores epidemiológicos e operacionais relacionados a doenças e agravos caracterizados como problema de saúde pública. Auxilia na elaboração de análises contextuais utilizadas na formulação de políticas e na avaliação de intervenções específicas no campo da saúde.

- Gestão/Financiamento: Aborda a execução orçamentária do MS por unidade orçamentária, programas e ações. Informa os limites financeiros por empenho e por fontes de recurso. Detalha a situação de convênios e apresenta as transferências fundo a fundo, particularizando os repasses 
financeiros. Exibe informações sobre estabelecimentos e profissionais de saúde em atuação no SUS. Mostra indicadores de processo e resultados do monitoramento do Pacto pela Saúde e, futuramente, do Contrato Organizativo de Ação Pública (COAP). Disponibiliza os dados referentes ao processo de gestão, à descentralização e ao controle social.

Além desses cinco módulos abertos, a Sage ainda disponibiliza a geração e impressão de relatórios gerenciais, com resumos estratégicos das informações, e a visualização de um menu interno, voltado para os gestores, com o monitoramento de ações, metas e indicadores estratégicos como os do Sistema de
Monitoramento de Obras (Sismob) e do programa SOS Emergências.

O SOS Emergências visa melhorar a gestão e a qualidade assistencial dos principais hospitais de urgência e emergência da rede SUS. Por conseguinte, a sala possibilita o monitoramento da evolução do programa de modo a estimular suas ações exitosas e a corrigir suas falhas. A Sage oferece, por exemplo, dados relativos a indicadores selecionados de morbimortalidade, que, por sua vez, possibilitam a realização de um recorte (municipal ou estadual) indicativo de motivos pelos quais os usuários procuram um ou outro hospital. Assim, os gestores que optam por aderir aos programas do MS podem traçar melhor seus planos de ação.

\section{- FIGURA 6: DASHBOARD DA SAGE COM INFORMAÇÕES SOBRE AS UNIDADES DE PRONTO ATENDIMENTO (UPA)}

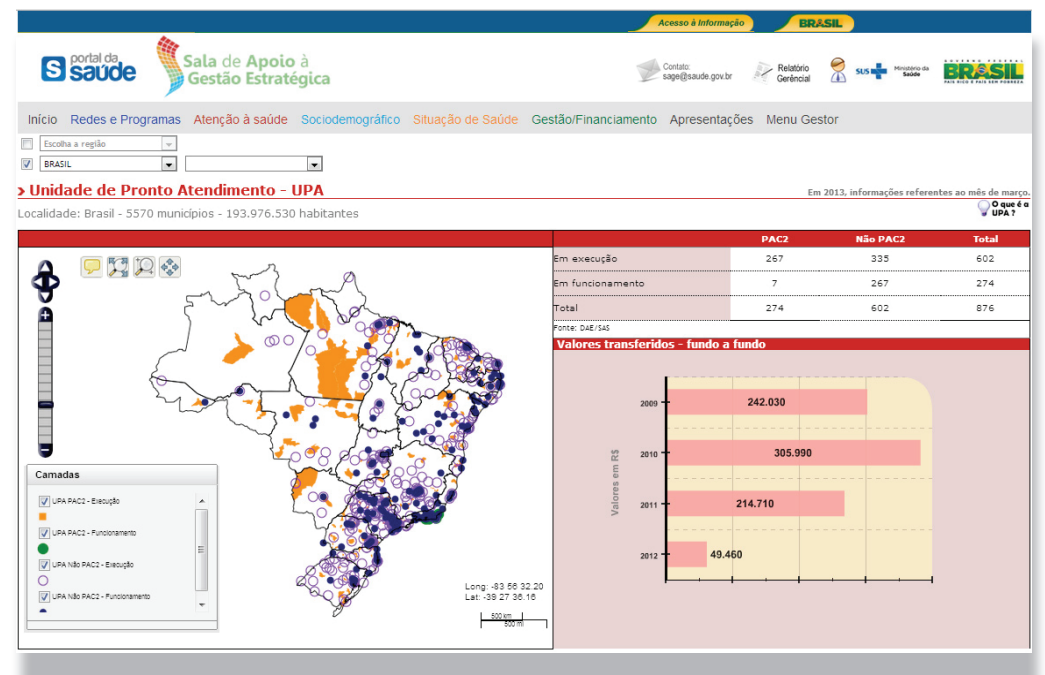

Fonte: Demas/SE/MS 


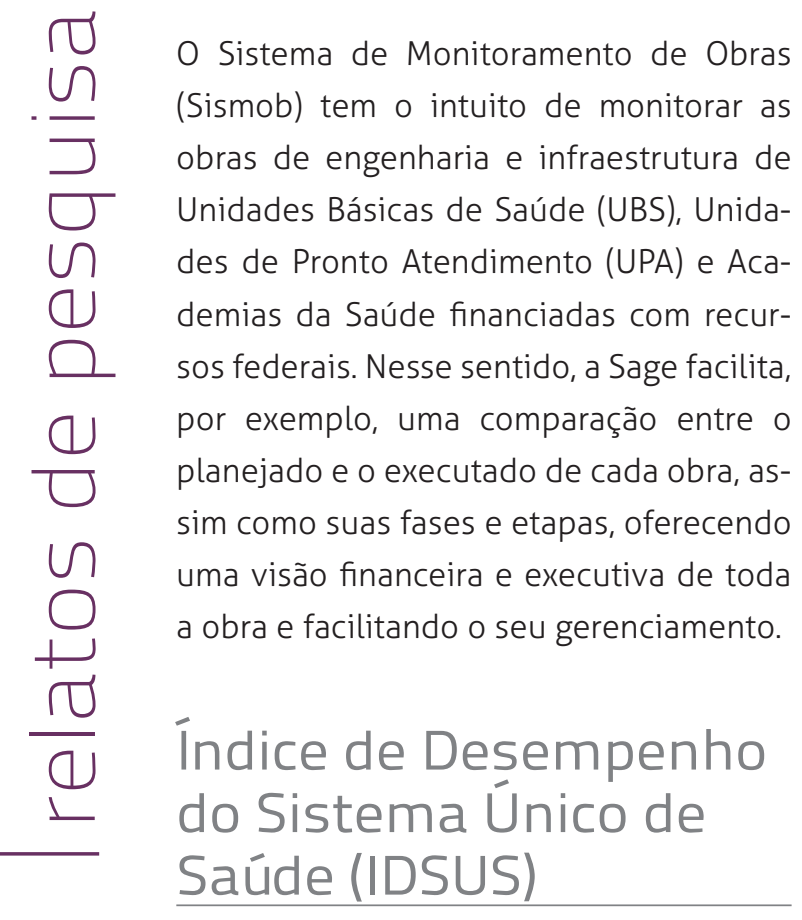

Desenvolvido pelo Demas a partir de um anseio explicitado no discurso de posse do Ministro da Saúde Alexandre Padilha, o Índice de Desempenho do SUS (IDSUS) visa avaliar o desempenho do sistema quanto ao cumprimento de seus princípios (universalidade do acesso, integralidade da atenção, equidade) e diretrizes (regionalização, hierarquização, comando único por esfera de gestão e responsabilidade tripartite) com base em indicadores calculados com dados dos Sistemas de Informações Nacionais ${ }^{1}$. Essa avaliação busca medir, aproximadamente, a distância entre o SUS existente e o SUS que atenderia a todos os brasileiros em todas suas necessidades de ações e serviços de saúde.

O núcleo elementar da avaliação realizada pelo IDSUS é o "SUS que atende os residentes de cada município brasileiro", pois contempla, além da Atenção Básica (realizada em todos os municípios), a Atenção Especializada, Ambulatorial e Hospitalar - tanto a realizada no próprio município, quanto a encaminhada para outros municípios, polos de uma região, de um estado ou nacional.

\section{- FIGURA 7: DASHBOARD DA SAGE COM INFORMAÇÕES DO SISTEMA DE MONITORAMENTO DE OBRAS (SISMOB)}

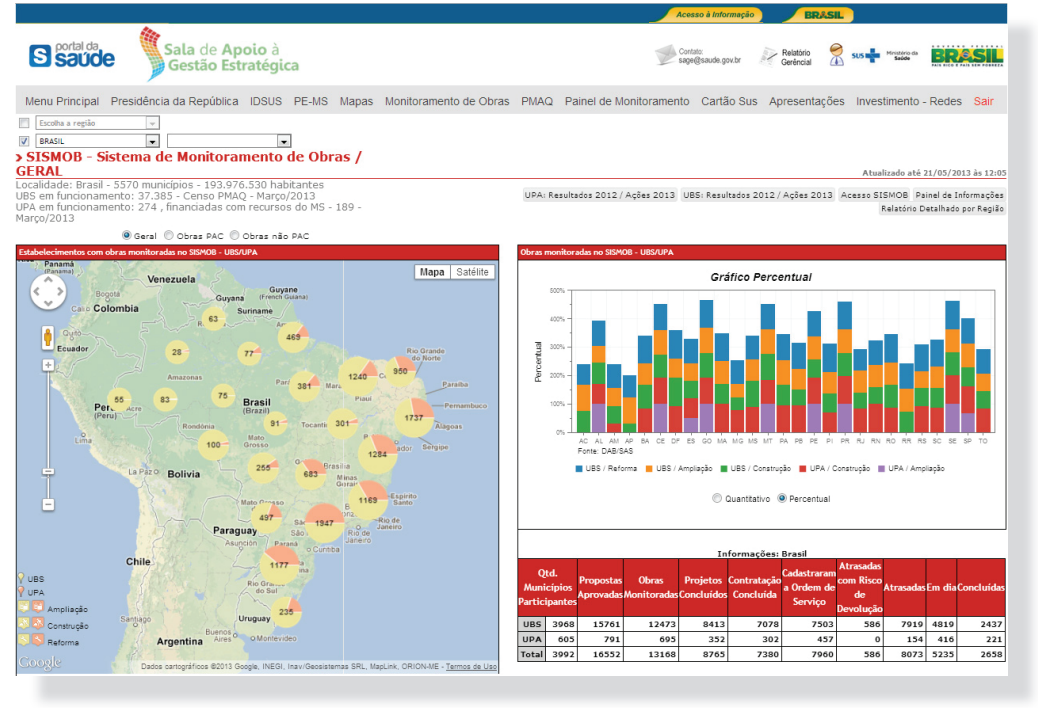


Em decorrência desse foco avaliativo, o IDSUS não avalia apenas isoladamente o sistema público de saúde existente nos territórios dos municípios, mas toda a rede pública, integrada e hierarquizada segundos os níveis de atenção, e até para além dessas. Isto é, ele avalia o conjunto de ações e serviços de saúde, organizado em níveis de complexidade crescente, com a finalidade de garantir a integralidade da assistência à saúde e sua articulação em uma Rede de Atenção à Saúde, conforme definido pelo Decreto n⿳0 7.508, de 28 de junho de 2011. Dessa forma, o IDSUS avalia o desempenho do Sistema Único de Saúde nos estados, regiões e no País por meio da média dos resultados da avaliação ponderada pelas respectivas populações ${ }^{2}$.

Em síntese, o IDSUS tem como finalidades: (i) avaliar o desempenho do SUS nos municípios, regionais de saúde, estados, regiões e no País; (ii) avaliar o acesso e a efetividade nos diferentes níveis de atenção: básica, especializada ambulatorial e hospitalar e urgências e emergências; (iii) expressar essa avaliação por meio de indicadores simples e compostos; (iv) fazer avaliação para detectar as deficiências, visando a melhorias e não apenas para classificar; e (v) fazer uma avaliação atrelada a um pacto de compromissos, pois os indicadores do IDSUS terão metas definidas no Contrato Organizativo de Ação Pública (COAP), que procura organizar as ações e serviços de saúde especializados em redes regionalizadas e hierarquizadas.

O Projeto Desenvolvimento de Metodologia de Avaliação do Desempenho do Sistema de Saúde Brasileiro (PRO-ADESS), da Associação Brasileira de Pós-Graduação em Saúde Coletiva (Abrasco), coordenado pelo Instituto de Comunicação e Informação Científica e Tecnológica em Saúde (ICICT) da Fundação Oswaldo Cruz (Fiocruz), serviu como referencial para o modelo de avaliação utilizado pelo IDSUS.

A partir disso, o IDSUS fixou como foco a avaliação do cuidado em saúde, por meio de indicadores capazes de mensurar o acesso potencial ou obtido e a efetividade das ações e serviços de saúde, regionalizados e realizados nos níveis da Rede de Atenção à Saúde, para os residentes de cada município brasileiro.

1 São fontes de dados dos indicadores do IDSUS: Cadastro Nacional de Estabelecimentos de Saúde (CNES); Sistema de Informação Ambulatorial (SIA); Sistema de Informação de Agravos de Notificação (SINAN); Sistema de Informação do Programa Nacional de Imunização (SI-PNI); Sistema de Informação Hospitalar (SIH); Sistema de Informação sobre Mortalidade (SIM); Sistema de Informação sobre Nascido Vivo (SINASC); Instituto Brasileiro de Geografia e Estatística (IBGE); Ministério do Desenvolvimento Social e Combate à Fome (MDS).

2 As notas, indicadores e mapas do Índice de Desempenho do Sistema Único de Saúde (IDSUS) são abertos e estão disponíveis para o acesso de todos no endereço: www.saude.gov.br/idsus.

Revista Brasileira de Monitoramento e Avaliação | Número 5 | Janeiro-Junho de 2013 
O modelo avaliativo do IDSUS conta ainda com um bloco de caracterização, segundo a homogeneidade dos municípios brasileiros, que foram agrupados em relação às semelhanças: socioeconômicas; do perfil de mortalidade infantil; e da estrutura do sistema de saúde existente no território municipal.

Baseado nas três dimensões do PRO-ADSESS (Determinantes da Saúde, Condições de Saúde da População, Estrutura do Sistema de Saúde), esse bloco, formado por seis grupos homogêneos, tem por objetivo fazer uma breve contextualização do desempenho encontrado no SUS que atende os residentes de cada município. Trata-se, portanto, de um recurso de análise dos resultados, não sendo utilizado para avaliar ou pontuar o desempenho do Sistema.

Para a construção do IDSUS foram selecionados 24 indicadores 3 distribuídos entre a atenção básica, as atenções ambulatorial e hospitalar e a urgência e emergência, compondo cinco índices. Além disso, a avaliação de desempenho do SUS efetuada por meio do IDSUS foi focada no cuidado na saúde, avaliando o acesso potencial ou obtido e a efetividade do cuidado na atenção básica, as atenções ambulatorial e hospitalar e a urgência e emergência.

\section{- OUADRO 1: MODELO AVALIATIVO DO ÍNDICE DE DESEMPENHO DO SUS (IDSUS)}

\begin{tabular}{|l|r|r|}
\hline \multicolumn{2}{|c|}{ DIMENSÕES PARA CARACTERIZAR OS MUNICÍPIOS } & RESULTADOS \\
\hline Determinantes da Saúde & Indicadores & \\
\hline Condições de Saúde da População & Indicadores & $\begin{array}{c}\text { Municípios segundo } \\
\text { extratos homogêneos }\end{array}$ \\
\hline Estrutura do Sistema de Saúde & Indicadores & \\
\hline & Porte Populacional & \\
\hline
\end{tabular}

\begin{tabular}{|c|c|c|}
\hline \multicolumn{3}{|c|}{ DESEMPENHO DO SUS QUE ATENDE OS RESIDENTES DOS MUNICÍPIOS } \\
\hline Indicadores de: & Nível de Atenção: & Por área: \\
\hline $\begin{array}{l}\text { Acesso } \\
\text { - Potencial ou Obtido } \\
\text { Efetividade } \\
\text { - Resultados esperados }\end{array}$ & $\begin{array}{l}\text { Atenção realizada no município } \\
\text { - Atenção básica ou primária } \\
\text { Atenção especializada realizada no município } \\
\text { e na rede de atenção à saúde } \\
\text { - Atenção ambulatorial geral e especializada } \\
\text { - Atenção hospitalar geral e especializada } \\
\text { - Urgência e Emergência }\end{array}$ & $\begin{array}{l}\text { - Saúde do adulto } \\
\text { - Saúde bucal } \\
\text { - Saúde da criança } \\
\text { - Saúde da mulher }\end{array}$ \\
\hline
\end{tabular}

\section{FORMA COMO OS RESULTADOS SÃO MOSTRADOS:}

Notas em cada nível da atenção e do desempenho geral do SUS que atende os residentes de cada município brasileiro, regiões, estados e nacional 
Para a avaliação, optou-se por utilizar uma metrificação conhecida. Dessa forma, as notas do IDSUS são quantificadas de 0 a 10. A intenção é que essa metrificação provoque questionamentos que possam mobilizar a população e gestores em prol de melhorias e na defesa do SUS.

De uma forma geral, os primeiros resultados do IDSUS apontaram que o maior problema do SUS ainda é o acesso e que a atenção básica, na maioria dos municípios brasileiros, está melhor do que a atenção especializada. Ele apontou que deve haver um aperfeiçoamento da regio- nalização, isto é, que deve ser melhorado o acesso dos moradores de cidades sem atenção especializada às cidades vizinhas, com mais infraestrutura de média e alta complexidade, ambulatorial e hospitalar, assim como aprimorado o acesso dos residentes desses municípios aos polos de referência.

O IDSUS apontou, ainda, os municípios que, além da atenção especializada, apresentam deficiências na atenção básica e que a deficiência da atenção especializada é maior nas regiões Norte, Nordeste e Centro-Oeste do que nas regiões Sudeste e Sul do Brasil.

\section{- OUADRO 2: INDICADORES ADOTADOS PARA COMPOR O IDSUS FONTE: DEMAS/SE/MS}

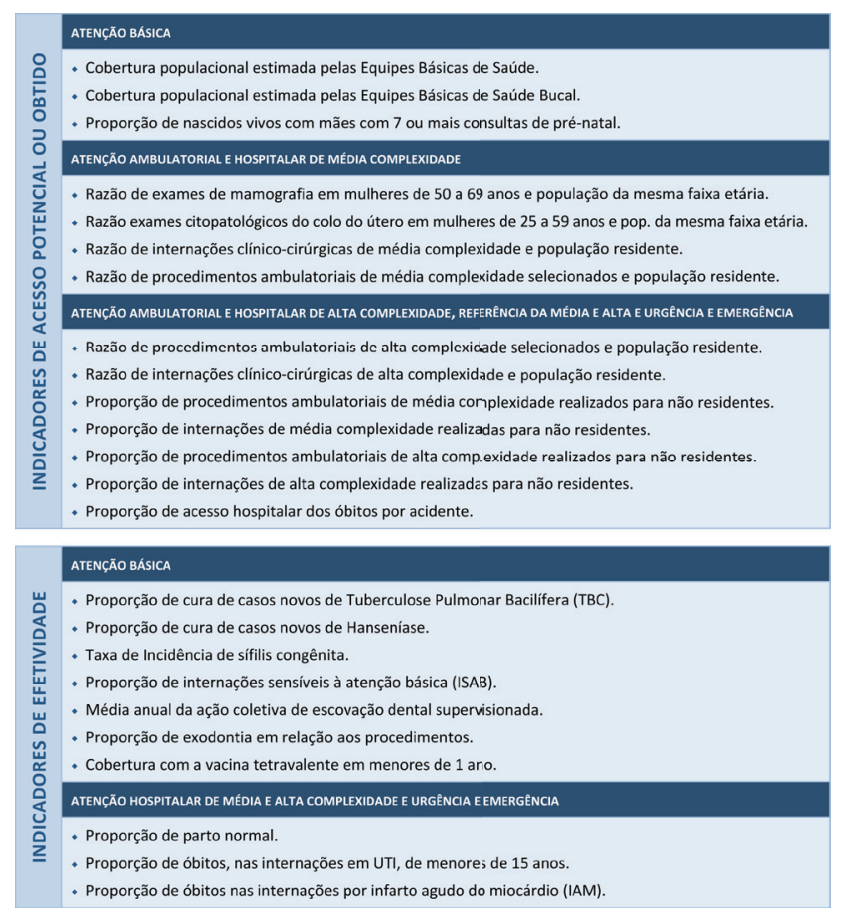

3 Foram submetidos a análises estatísticas diversos indicadores, visando identificar imprecisões. Só foram utilizados aqueles que apresentaram maior grau de consistência e fidedignidade. Assim, foram testados vários indicadores, sendo selecionados aqueles que se mostraram mais confiáveis, relevantes e calculáveis para todos os municípios brasileiros.

Revista Brasileira de Monitoramento e Avaliação | Número 5 | Janeiro-Junho de 2013 
A leitura dos resultados do IDSUS permite mapear quais são os pontos fortes e fragilidades de cada município, nos indicadores simples e compostos. A análise dos resultados dos indicadores simples do SUS, em municípios de uma mesma região de saúde, permite o refinamento do olhar para as diferenças entre os SUS que atendem os residentes desses municípios, seus pontos positivos e negativos. Ambas as visões podem subsidiar os gestores federal, estaduais e municipais no estabelecimento das prioridades de ações visando a melhorias.

Mais que um ranking e menos que uma explicação exaustiva, o IDSUS oferece uma visão aproximada do SUS, que possibilita a instauração de práticas de avaliação e monitoramento para identificar os principais problemas do Sistema e, principalmente, para repor a necessidade de pactos entre os gestores, com metas e compromissos para a contínua construção de um SUS que responda mais aos direitos de cidadania e de saúde do povo brasileiro.

Juntamente com outros componentes de avaliação e monitoramento (como o Programa Nacional de Avaliação dos Serviços de Saúde (PNASS); as pesquisas de avaliação do acesso aos serviços e satisfação dos usuários; o Programa Nacional de Melhoria do Acesso e da Qualidade da Atenção Básica (PMAO); e o Programa de Avaliação da Gestão do SUS), o IDSUS se consolida como uma das partes integrantes de um complexo sistema de planejamento, monitoramento e avaliação que servirá de base para a construção da Política de Monitoramento e Avaliação e do Sistema de Avaliação do SUS brasileiro. 


\section{- FIGURA 8: REPRESENTAÇÃO GRÁFICA DA COMPOSIÇÃO DOS INDICADORES QUUE FORMAM O IDSUS}

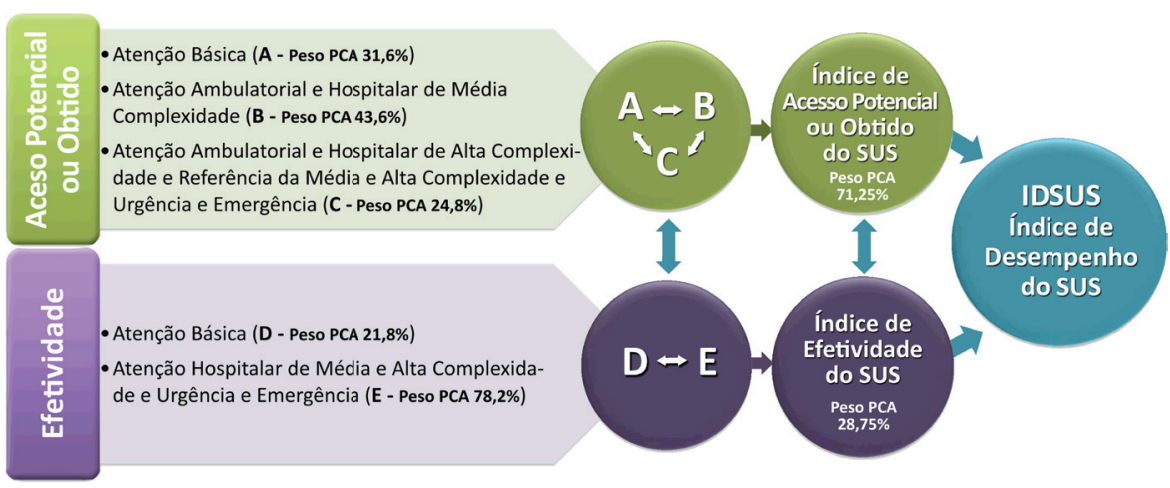

Fonte: Demas/SE/MS 